\title{
Incidence And Risk Factors of Hypertension Therapy In Australian Cancer Patients Treated With Vascular Signalling Pathway Inhibitors
}

Soojung Hong ( $\square$ suzzy901@nhimc.or.kr)

Centre for Big Data Research in Health, UNSW Sydney

Benjamin Daniels

Centre for Big Data Research in Health, UNSW Sydney

Marina T. Leeuwen

Centre for Big Data Research in Health, UNSW Sydney

Sallie-Anne Pearson

Centre for Big Data Research in Health, UNSW Sydney

Claire M. Vajdic

Centre for Big Data Research in Health, UNSW Sydney

\section{Research Article}

Keywords: cancer, hypertension, vascular signalling pathway inhibitors, incidence, risk factors

Posted Date: October 26th, 2021

DOI: https://doi.org/10.21203/rs.3.rs-1006781/v1

License: (9) This work is licensed under a Creative Commons Attribution 4.0 International License.

Read Full License

Version of Record: A version of this preprint was published at Discover Oncology on January 20th, 2022.

See the published version at https://doi.org/10.1007/s12672-022-00468-3. 


\section{Abstract}

Background Clinical trials report systemic hypertension is an adverse effect of vascular signalling pathway inhibitor (VSPi) use. There are limited data from routine clinical practice. We aimed to estimate the real-world incidence and risk factors of new-onset and aggravated hypertension for cancer patients dispensed VSPi in whole-of-population Australian setting.

Methods We used dispensing records for a $10 \%$ random sample of Australians to identify treatment with subsidised VSPi from 2013-2018. We further identified dispensings of oral antihypertensive medicines 6 months before and during VSPi therapy. We defined (i) new-onset hypertension in people first dispensed antihypertensives after VSPi and (ii) aggravated hypertension in people with prior antihypertensive use dispensed an additional, or higher strength, antihypertensive after VSPi. We applied the Fine-Gray cumulative incidence function and Cox proportional hazard regression.

Results 1,802 patients were dispensed at least one VSPi. The mean age of the cohort was 65 years and $57 \%$ were male. The incidence of new-onset treated hypertension was $24.3 \%$ (95\% Cl: 21.2-27.8); age ${ }^{3} 60$ years (HR 1.74; 95\% Cl: 1.32-2.31) and treatment with oral tyrosine kinase inhibitors compared to bevacizumab (HR 1.96; 95\% Cl: 1.16-3.31) were risk factors. The incidence of aggravated hypertension was $25.2 \%$ (95\% Cl: 22.0-28.7) and risk was elevated for patients with renal cancer (HR 2.84; 95\% Cl: 1.49$5.41)$ and cancers other than colorectal (HR 1.85; 95\% Cl: 1.12-3.03).

Conclusions Our real-world estimates of incident hypertension appear comparable to those observed in clinical trials (21.6-23.6\%). Our population-based study provides some insight into the burden of hypertension in patients commencing VSPi in routine practice.

\section{Introduction}

Angiogenesis has an important role in tumour cell proliferation and metastasis, making the vascular endothelial growth factor (VEGF) signalling pathway (VSP) a critical therapeutic target of cancer therapy [1]. Several medicines targeting the VSP are available for cancer treatment and VSP inhibitors (VSPi) are currently one of the most common medicine classes used in the treatment of solid cancers $[2,3]$. These agents also target normal cardiovascular physiology, known as an 'on-target' effect [4]. This can lead to adverse cardiovascular effects including hypertension, thrombosis, heart failure and stroke $[5,6]$. Systemic hypertension, the most common cardiotoxicity in patients using VSPi, results from increased vascular resistance and systemic thrombotic microangiopathy [7]. The incidence of hypertension associated with bevacizumab, the most common VSPi, is $23.6 \%$ in clinical trials [8-10]. The incidence of VSPi-induced hypertension from other meta-analyses range from $21.6 \%$ with sunitinib [11] to $47 \%$ with lenvatinib [12]. If not properly managed, hypertension can cause end organ damage to the heart, kidney, and brain [13]. In addition, if it is not controlled or complications occur, the VSPi could be discontinued, potentially impairing cancer control and shortening patient survival. 
All meta-estimates for VSPi-induced hypertension are based on phase II/III clinical trials, where patients are generally highly selected, younger and with fewer comorbidities than typical cancer patients. Currently, there are limited real-world data and no whole-of-population estimates of new onset and aggravated hypertension in VSPi treated patients. A United States' (US) claims-based study of commercially insured patients reported new-onset hypertension in $32 \%$ of a cohort of approximately 1000 cancer patients receiving VSPi [14]. Another US observational study based on electronic medical records from a single health care network found $50 \%$ of approximately 1100 patients had VSPi-induced hypertension, with elevated risk for those with pre-existing hypertension, age $\geq 60$ years, and higher body mass index [15]. Both of these studies defined hypertension occurrence as a diagnostic code for hypertension or a pharmacy claim for an antihypertensive. Here we describe the real-world incidence and risk factors of new-onset and aggravated hypertension for patients dispensed VSPi in a whole-ofpopulation setting in Australia.

\section{Methods}

Study design

A population-based, retrospective cohort study.

Data source and study population

All Australian citizens and permanent residents are entitled to subsidised prescribed medicines through the Pharmaceutical Benefit Scheme (PBS). We used dispensing records for a $10 \%$ random sample of PBS-eligible Australians. The $10 \%$ PBS sample is a routine dataset provided by Services Australia for analytical use; people are selected based on the last digit of a randomly assigned unique identifier. This dataset captures all dispensed PBS-listed medicines, including PBS medicines not attracting a subsidy, from 1 July 2012 [16]. To protect the privacy of people in this dataset, all dates of dispensing are offset randomly by $+/-14$ days; the direction of the offset is the same for all records for each individual [17].

Our study population included all adults (aged $\geq 18$ years) with a new dispensing record for a VSPi between 1 January 2013 and 30 September 2018. We defined the index treatment date as the first date of VSPi dispensing in this period. Allowing for a 180 day look-back period and at least one year follow-up, the study period was dispensing records from 1 July 2012 to 30 September 2019. We excluded patients with less than one-month follow-up and those with a single dispensing claim.

Medicines of interest

We identified all PBS-subsidised VSPi medicines in our sample; bevacizumab, sunitinib, sorafenib, pazopanib, axitinib and lenvatinib. Bevacizumab is a monoclonal antibody administered intravenously on a 2- or 3-week schedule for colorectal (CRC), cervical, and ovarian cancer. The other agents are small molecule tyrosine kinase inhibitors (TKIs) administered daily orally, with one prescription lasting 30 days. In Australia, sunitinib is indicated for pancreatic neuroendocrine tumours (pNET), malignant 
gastrointestinal stromal tumours (GIST), and renal cell carcinoma (RCC). Sorafenib is indicated for RCC and hepatocellular carcinoma (HCC), pazopanib for RCC and soft tissue sarcoma (STS), axitinib for RCC, and lenvatinib for thyroid cancer [18]. We determined primary cancer site based on the VSPi PBS item code dispensed to each patient.

We classified dispensings of PBS-listed oral antihypertensive medicines using the Anatomical Therapeutic Chemical (ATC) Classification System: C03 Diuretics, C07 Beta-blockers, C08 Calcium channel blockers, and C09 Agents acting on the renin-angiotensin system including fixed-dose combinations. We excluded the $\mathrm{CO} 2$ (antihypertensives) class because these treatments are more commonly used to treat conditions other than hypertension in Australia [19]. The dispensing of hypertensive medications was used to identify pre-existing hypertension and also used for our outcome measure.

\section{Measures}

We calculated the duration of oral VSPi medicine as the first to the last dispensing date plus the dispensed quantity (30 days). For bevacizumab, the duration of use was calculated as the first to the last dispensing date plus 14 days because more than $90 \%$ of patients received this medicine every 2-weeks.

We classified people with at least one antihypertensive dispensing record in the 180 days prior to the date of first VSPi dispensing as having pre-existing pharmacologically treated hypertension. All other patients were classified as having no prior hypertension treatment. We defined new-onset hypertension as people first dispensed an antihypertensive whilst being dispensed a VSPi. We defined the aggravated hypertension group as those patients with pre-existing hypertension who were dispensed an additional, or higher tablet strength (escalated dose same or replacement/switched agent), antihypertensive whilst being dispensed a VSPi. We identified other comorbidities (excluding malignancy and hypertension) using the Rx-risk comorbidity score applied to dispensing records for the six months prior to commencing VSPi treatment [20]. The Rx-risk is a measure of an individual's current comorbidities based on their dispensing history. It has been shown to predict 1-year mortality in multiple prescription datasets, including the 10\% PBS sample [20]. Each identified comorbidity was given a score of one, and the scores were summed for each individual to derive an overall comorbidities score.

\section{Statistical analysis}

All patients were followed from the index date until the first dispensing or change in antihypertensive medicine, the end of VSPi treatment, death, or the end of follow up, whichever came first.

We defined the incidence rate as the number of patients who developed new onset or aggravated hypertension during follow-up divided by the total person-time of observation. We presented these as events per 1000 person-years and $95 \%$ confidence intervals $(95 \% \mathrm{Cl})$. We considered death as a competing risk for the time to event analyses. The dataset provided the fact and year of death; we defined the date of death as the last date of any dispensed medicines plus 90 days [21]. We calculated the 
incidence of hypertension (new onset and aggravated) overall and by sex, age, comorbidity, type of cancer, and type of VSPi using the Fine and Gray cumulative incidence function [22].

We compared the baseline characteristics of people with 'no prior treated hypertension' and 'pre-existing treated hypertension' using the Chi-square test. We described the prescribing pattern of antihypertensive medicines including the first-line therapy and the number of antihypertensive medicines. We compared the median time from VSPi dispensing to first dispensing of an antihypertensive agent for patients treated with oral TKIs and bevacizumab using quantile regression. To evaluate risk factors for developing new onset or aggravated hypertension, we used cause-specific Cox proportional hazard regression to estimate hazard ratios (HR) and $95 \% \mathrm{Cls}$. We examined the association with all available putative risk factors, specifically age, sex, comorbidity, cancer type, and type of VSPi. All statistical analyses were conducted using SAS version 9.4 (SAS Institute, Cary NC).

\section{Results}

\section{Cohort characteristics}

We identified 1802 patients fulfilling our criteria for VSPi use (Fig. 1), 906 (50.3\%) of whom had preexisting treated hypertension. The mean age of the cohort was 65.1 years (SD 12.5); people with preexisting treated hypertension (66.0 years) were older than those with no prior hypertension (60.1 years) (Table 1). Overall, there were more males than females ( $57.5 \%$ vs. $42.5 \%$ ), and a higher proportion of males with the pre-existing hypertension. The mean comorbidity score was 3.2 (SD 2.1) for all patients, and the comorbidity burden was higher in the pre-existing compared to the no prior hypertension group. The most common cancer was CRC (58.2\%), followed by RCC (14.8\%) and HCC (12.3\%). 
Table 1

Study population dispensed VSPi $(n=1,802)$

\begin{tabular}{|c|c|c|c|c|c|c|c|}
\hline \multirow[t]{2}{*}{ Characteristic } & \multicolumn{2}{|c|}{$\begin{array}{l}\text { Overall } \\
(n=1,802)\end{array}$} & \multicolumn{2}{|c|}{ No prior HTN $(n=896)$} & \multicolumn{2}{|c|}{ Prior HTN (n=906) } & \multirow[t]{2}{*}{ P-value } \\
\hline & $\mathbf{n}$ & $\%$ & $\mathbf{n}$ & $\%$ & $\mathbf{n}$ & $\%$ & \\
\hline Mean age (years) & \multicolumn{2}{|c|}{65.1 (SD 12.5) } & \multicolumn{2}{|c|}{60.1 (SD 12.7) } & \multicolumn{2}{|c|}{66.0 (SD 10.2) } & $<0.01$ \\
\hline \multicolumn{8}{|l|}{ Sex } \\
\hline Male & 1,036 & 57.5 & 470 & 52.5 & 566 & 62.5 & $<0.01$ \\
\hline Female & 766 & 42.5 & 426 & 47.5 & 340 & 37.5 & \\
\hline \multicolumn{8}{|c|}{ Number of comorbid conditions (Rx-risk) } \\
\hline Mean & \multicolumn{2}{|c|}{3.2 (SD 2.1) } & \multicolumn{2}{|c|}{2.5 (SD 1.8) } & \multicolumn{2}{|c|}{3.9 (SD 2.2) } & $<0.01$ \\
\hline 0 & 143 & 7.9 & 116 & 13.0 & 27 & 3.0 & $<0.01$ \\
\hline $1-2$ & 591 & 32.8 & 366 & 40.9 & 225 & 24.8 & \\
\hline $3-4$ & 611 & 33.9 & 288 & 32.1 & 323 & 35.7 & \\
\hline$\geq 5$ & 457 & 25.4 & 126 & 14.1 & 331 & 36.5 & \\
\hline \multicolumn{8}{|l|}{ Cancer type } \\
\hline Colorectal & 1,049 & 58.2 & 560 & 62.5 & 489 & 54.0 & $<0.01$ \\
\hline Renal & 267 & 14.8 & 114 & 12.7 & 153 & 16.9 & \\
\hline Liver & 222 & 12.3 & 60 & 6.7 & 162 & 17.9 & \\
\hline Ovarian & 134 & 7.4 & 84 & 9.4 & 50 & 5.5 & \\
\hline Sarcoma & 55 & 3.1 & 36 & 4.0 & 19 & 2.1 & \\
\hline GIST & 26 & 1.4 & 14 & 1.6 & 12 & 1.3 & \\
\hline Thyroid & 20 & 1.1 & 7 & 0.8 & 13 & 1.4 & \\
\hline Cervix & 20 & 1.1 & 16 & 1.6 & 4 & 0.4 & \\
\hline Pancreatic NET & 9 & 0.5 & 5 & 0.6 & 4 & 0.4 & \\
\hline \multicolumn{8}{|l|}{ VSPi agent } \\
\hline Bevacizumab & 1,203 & 66.8 & 660 & 73.7 & 543 & 59.9 & $<0.01$ \\
\hline
\end{tabular}

EGFR, epidermal growth factor receptor; Ab, antibody; HTN, hypertension; GIST, gastrointestinal stromal tumour; NET, neuroendocrine tumour

a Less than 3; suppressed to protect patient confidentiality 


\begin{tabular}{|c|c|c|c|c|c|c|c|}
\hline \multirow[t]{2}{*}{ Characteristic } & \multicolumn{2}{|c|}{$\begin{array}{l}\text { Overall } \\
(n=1,802)\end{array}$} & \multicolumn{2}{|c|}{ No prior HTN $(n=896)$} & \multicolumn{2}{|c|}{ Prior HTN $(n=906)$} & \multirow[t]{2}{*}{ P-value } \\
\hline & $\mathbf{n}$ & $\%$ & $\mathbf{n}$ & $\%$ & $\mathbf{n}$ & $\%$ & \\
\hline Sorafenib & 234 & 13.0 & 66 & 7.4 & 168 & 18.5 & \\
\hline Pazopanib & 218 & 12.1 & 104 & 11.6 & 114 & 12.6 & \\
\hline Sunitinib & 126 & 7.0 & 59 & 6.6 & 67 & 7.4 & \\
\hline Lenvatinib & 20 & 1.1 & 7 & 0.8 & 13 & 1.4 & \\
\hline Axitinib & a & 0 & a & 0 & a & 0.1 & \\
\hline \multicolumn{8}{|c|}{ Prior chemotherapy (6 months prior to VSPi) } \\
\hline No & 881 & 48.9 & 394 & 44.03 & 487 & 53.8 & $<0.01$ \\
\hline Yes & 921 & 51.1 & 502 & 56.0 & 419 & 46.3 & \\
\hline \multicolumn{8}{|c|}{ Type of prior chemotherapy } \\
\hline Fluorouracil & 725 & 44.4 & 376 & 41.5 & 349 & 48.0 & 0.02 \\
\hline Platinum & 526 & 32.2 & 289 & 31.9 & 237 & 32.6 & \\
\hline Irinotecan & 125 & 7.7 & 75 & 8.3 & 50 & 6.9 & \\
\hline Taxane & 110 & 6.7 & 71 & 7.8 & 39 & 5.4 & \\
\hline Anti-EGFR Ab & 38 & 2.3 & 25 & 2.8 & 13 & 1.8 & \\
\hline Anthracycline & 35 & 2.1 & 25 & 2.8 & 10 & 1.4 & \\
\hline Other & 75 & 4.6 & 46 & 5.1 & 29 & 4.0 & \\
\hline \multicolumn{8}{|c|}{$\begin{array}{l}\text { EGFR, epidermal growth factor receptor; Ab, antibody; HTN, hypertension; GIST, gastrointestinal } \\
\text { stromal tumour; NET, neuroendocrine tumour }\end{array}$} \\
\hline
\end{tabular}

Bevacizumab was the most frequently used VSPi ( $66.8 \%$ of all patients), and the rate of use was higher in people who had not been treated previously for hypertension compared with those previously treated (73.7\% vs $59.9 \%)$. Among oral TKIs, the most frequently prescribed medicine was sorafenib in people who had pre-existing hypertension and pazopanib in people who had no prior hypertension. About half the patients (48.9\%) were dispensed VSPi as the first line treatment for this therapeutic stage of disease. The remainder most frequently received fluorouracil (5-FU and capecitabine) and platinum (cisplatin, carboplatin, and oxaliplatin) classes in the 6 months prior to using VSPi. Patients were treated with VSPi for a median of 206 days (range 15-2176) and the duration was not significantly different between the two groups (226 days in no prior hypertension group vs. 197 days in pre-existing hypertension group). 
The total duration of follow-up was 1194 person-years, and the average was 0.66 person-years (SD 0.79 ) or 241 days. For the 896 people without a history of treated hypertension, new-onset hypertension occurred during VSPi use in 218 patients (Table 2$)$. The overall incidence was $24.3 \%$ (95\% Cl, 21.2-27.8) and the incidence rate was $352.8 / 1000$ person-years $(95 \% \mathrm{Cl}, 308.9-402.9)$. The median time from VSPi dispensing to first dispensing of an antihypertensive agent was 78 days (range 4-945); significantly shorter for patients treated with oral TKIs than bevacizumab (median 53 vs 95 days). Most (49\%) people with new-onset hypertension were initially prescribed a single antihypertensive agent; most commonly angiotensin-converting enzyme (ACE) inhibitors or angiotensin receptor blockers (ARBs) $(47.8 \%, N=106)$. Fifty-nine patients (26.6\%) initially received a calcium channel blocker (CCB); only six patients were dispensed combination agents at the time of treatment initiation. 
Table 2

Characteristics of VSPi cohort subgroups with and without pre-existing hypertension

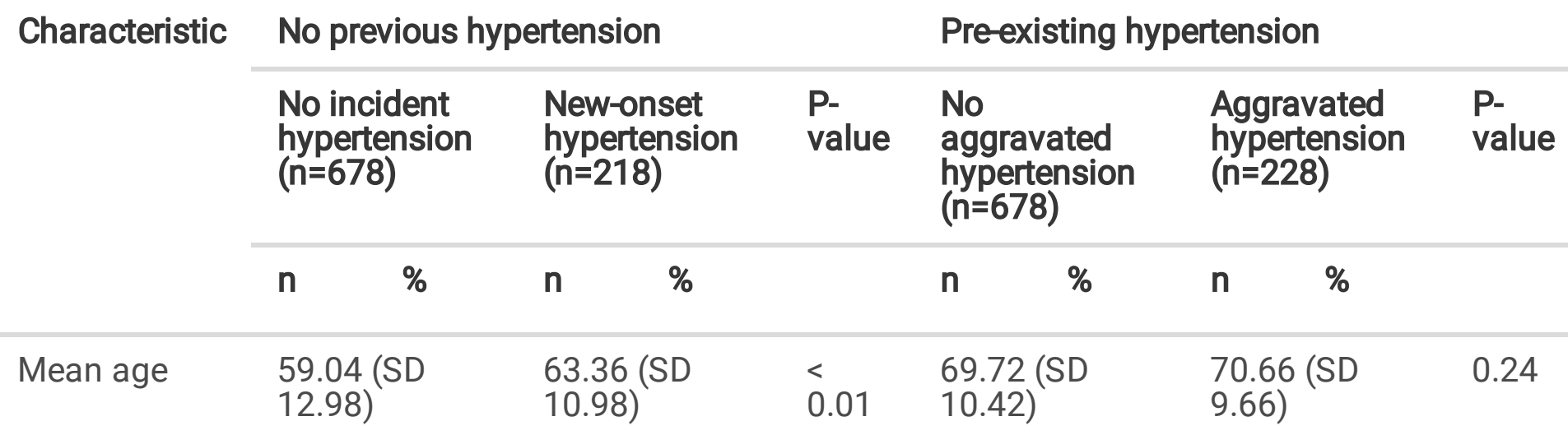

Sex

$\begin{array}{lllllllllll}\text { Male } & 352 & 51.9 & 118 & 54.1 & 0.57 & 431 & 63.6 & 135 & 59.21 & 0.43\end{array}$

$\begin{array}{lllllllll}\text { Female } & 326 & 48.1 & 100 & 45.9 & 247 & 36.4 & 93 & 40.79\end{array}$

Number of comorbid conditions (Rx-Risk)

$\begin{array}{lllllllllll}\text { Mean } & 2.5(\text { SD 1.8) } & 2.4(\text { SD 1.8) } & 0.45 & 4.0(\text { SD 2.2) } & 3.8 \text { (SD 2.2) } & 0.23 \\ 0 & 85 & 12.5 & 31 & 14.2 & 0.66 & 19 & 2.8 & 8 & 3.5 & 0.26 \\ 1-2 & 275 & 40.6 & 91 & 41.7 & & 158 & 23.3 & 67 & 29.4 & \\ 3-4 & 225 & 33.2 & 63 & 28.9 & 249 & 36.7 & 74 & 32.5 & \\ \geq 5 & 93 & 13.7 & 33 & 15.1 & & 252 & 37.2 & 79 & 34.7 & \end{array}$

Cancer type

$\begin{array}{lllllllllll}\text { Colorectal } & 447 & 65.9 & 113 & 51.8 & < & 377 & 55.6 & 112 & 49.1 & 0.09\end{array}$

$\begin{array}{lllllllll}\text { Renal } & 74 & 10.9 & 40 & 18.4 & 104 & 15.3 & 49 & 21.5 \\ \text { Liver } & 39 & 5.8 & 21 & 9.6 & 127 & 18.7 & 35 & 15.4\end{array}$

$\begin{array}{lllllllll}\text { Ovarian } & 59 & 8.7 & 25 & 11.5 & 31 & 4.6 & 19 & 8.3\end{array}$

$\begin{array}{lllllllll}\text { Sarcoma } & 28 & 4.1 & 8 & 3.7 & 15 & 2.2 & 4 & 1.8\end{array}$

$\begin{array}{lllllllll}\text { GIST } & 6 & 0.9 & 8 & 3.7 & 9 & 1.3 & 3 & 1.3 \\ \text { Others } & 25 & 3.7 & 3 & 1.4 & 15 & 2.2 & 6 & 2.6\end{array}$

VSPi

$\begin{array}{lllllllllll}\text { Bevacizumab } & 521 & 76.8 & 139 & 63.8 & < & 411 & 60.6 & 132 & 57.9 & 0.47\end{array}$ 


\begin{tabular}{|c|c|c|c|c|c|c|c|c|c|c|}
\hline \multirow[t]{3}{*}{ Characteristic } & \multicolumn{5}{|c|}{ No previous hypertension } & \multicolumn{5}{|c|}{ Preexisting hypertension } \\
\hline & \multicolumn{2}{|c|}{$\begin{array}{l}\text { No incident } \\
\text { hypertension } \\
(n=678)\end{array}$} & \multicolumn{2}{|c|}{$\begin{array}{l}\text { New-onset } \\
\text { hypertension } \\
(n=218)\end{array}$} & \multirow[t]{2}{*}{$\begin{array}{l}\mathrm{P} \text { - } \\
\text { value }\end{array}$} & \multicolumn{2}{|c|}{$\begin{array}{l}\text { No } \\
\text { aggravated } \\
\text { hypertension } \\
(n=678)\end{array}$} & \multicolumn{2}{|c|}{$\begin{array}{l}\text { Aggravated } \\
\text { hypertension } \\
(n=228)\end{array}$} & \multirow[t]{2}{*}{$\begin{array}{l}\mathrm{P}- \\
\text { value }\end{array}$} \\
\hline & $\mathbf{n}$ & $\%$ & $\mathrm{n}$ & $\%$ & & $\mathrm{n}$ & $\%$ & $\mathrm{n}$ & $\%$ & \\
\hline TKI & 157 & 23.2 & 79 & 36.2 & & 267 & 39.4 & 96 & 42.1 & \\
\hline
\end{tabular}

A total of 906 patients had hypertension prior to VSPi therapy. ACE inhibitors or ARB were the most frequently prescribed (32.4\%), followed by combination agents (19.1\%), beta-blockers $(18.1 \%)$, CCB (15.3\%), and diuretics (15.1\%). Among them, 228 patients experienced aggravated hypertension requiring an additional, or higher tablet strength, antihypertensive. The overall incidence of aggravated hypertension was $25.2 \%(95 \% \mathrm{Cl}, 22.0-28.6)$ and the incidence rate was $395.6 / 1000$ person-years $(95 \% \mathrm{Cl}$, 347.5-450.4). The median time from the first VSPi dispensing to aggravation was 70 days (range 21806). Patients with aggravated hypertension were dispensed an average of two antihypertensive agents before aggravation and an average of three agents after aggravation. After aggravation, ACE inhibitors, ARBs and combination ACE/ARB agents were dispensed less often, and CCBs and diuretics were dispensed more often.

The cumulative incidence of new onset and aggravated hypertension was not statistically different (Fig. 2).

Hypertension risk factors

In univariable analyses, age $\geq 60$ years, cancer type other than CRC, and oral TKIs were significantly associated with new-onset hypertension. In multivariable analysis, age $>60$ years $(\mathrm{HR}, 1.74 ; 95 \% \mathrm{Cl}, 1.32-$ 2.31) and treatment with oral TKI agents $(\mathrm{HR}, 1.96 ; 95 \% \mathrm{Cl}, 1.16-3.31)$ were independent risk factors (Table 3). 
Table 3

Risk factors for new-onset and aggravated hypertension after VSPi in multivariable analysis

\begin{tabular}{|lllll|}
\hline Characteristic & \multicolumn{2}{l}{$\begin{array}{l}\text { New onset } \\
\text { hypertension }\end{array}$} & \multicolumn{3}{l|}{$\begin{array}{l}\text { Aggravated } \\
\text { hypertension }\end{array}$} \\
\cline { 2 - 6 } & HR & $95 \%$ Cl & HR & $95 \%$ Cl \\
\hline Age $>60$ years & 1.74 & $1.32-2.31$ & 1.26 & $0.86-1.84$ \\
\hline Female sex & 1.10 & $0.82-1.48$ & 1.09 & $0.82-1.44$ \\
\hline $\begin{array}{l}\text { Higher than median number of comorbid } \\
\text { conditions }{ }^{2}\end{array}$ & 0.97 & $0.74-1.28$ & 0.87 & $0.67-1.13$ \\
\hline Cancer type & & & & \\
\hline RCC (vs. CRC) & 1.22 & $0.66-2.27$ & 2.84 & $1.49-5.41$ \\
\hline Others (vs. CRC) & 1.36 & $0.87-2.12$ & 1.85 & $1.12-3.04$ \\
\hline Oral TKI (vs. Bevacizumab) & 1.96 & $1.16-3.31$ & 0.68 & $0.39-1.19$ \\
\hline CRC, colorectal cancer; HR, hazard ratio; RCC, renal cell carcinoma; TKI, tyrosine kinase inhibitor & \\
\hline a the median number of comorbid conditions was 3.0 & & & \\
\hline
\end{tabular}

Risk of aggravated hypertension was associated with cancer type other than CRC, and oral TKIs in univariable analyses. In multivariable analysis, only cancer type was an independent risk factor; compared to CRC, risk was elevated for RCC (HR, 2.84; $95 \% \mathrm{Cl}, 1.49-5.41)$ and other cancer types (HR 1.85; 95\% Cl, 1.12-3.03) (Table 3).

\section{Discussion}

In our real-world study, the overall incidence of new-onset and aggravated hypertension during VSPi treatment was similar, at $24.3 \%$ and $25.2 \%$ respectively. These estimates are lower than reported in other observational studies and slightly higher than meta-estimates summarising the clinical trial experience where hypertension was measured [10-12,23-25]. In clinical trial meta-analyses, the incidence of VSPiinduced hypertension (any grade) was $23.6 \%$ for bevacizumab [10], 23.1\% for sorafenib [23], and 21.6\% for sunitinib [11]. Cancer patients participating in randomized clinical trials are generally highly selected and in otherwise good health, and thus adverse events are observed less frequently than in real-world practice [26]. The two previous US claims-based studies, using International Classification of Diseases (ICD) codes for hypertension or codes for dispensed anti-hypertensive medicines $[14,15]$ observed higher incidence estimates, at $32 \%$ and $49 \%$ respectively. Our lower estimates may reflect the fact that we could only identify patients dispensed antihypertensives, not all those diagnosed with the condition. In one US real-world clinical practice study, $14.1 \%$ of patients with newly diagnosed hypertension during VSPi use were not treated with antihypertensives [14]. In a recent large pooled cohort study of Australian and New Zealand adults who were diagnosed with cancer during follow-up, 33\% had untreated hypertension and 
$25 \%$ had treated hypertension at baseline [27]. Hypertension was ascertained via blood pressure measurements and treatment was based on self-report.

For patients with new-onset hypertension, the first antihypertensive dispensing was a median of 78 days from the index date, similar to a previous US claims-based analysis (96 days) [14]. The time interval to hypertension treatment differed by type of VSPi, and was significantly longer for patients treated with bevacizumab than oral TKIs. Among the oral TKIs, the shortest time interval was observed with pazopanib. VSPi-induced hypertension has been shown to develop rapidly, and to return to baseline after drug withdrawal $[4,28,29]$. Studies monitoring blood pressure have revealed blood pressure increases within hours to days after initiating oral TKIs $[28,29]$. In one such study, the median time to first documented hypertensive response was 29 days in VSP-TKI treated patients [15].

For patients with new-onset VSPi-induced hypertension, the current guidelines recommend medications targeting the renin-angiotensin-aldosterone (RAAS) pathway such as ACE inhibitors, ARBs, and dihydropyridine CCBs as first-line therapy [30-32]. Most of the patients in our new-onset hypertension group were dispensed ACE inhibitors, ARBs, and CCBs and half started with monotherapy.

We identified patients with pre-existing hypertension based on the dispensing of an antihypertensive agent during the 6 months prior to starting VSPi treatment. Over $50 \%$ of our cohort met this criteria, consistent with US claims data [14]. These patients were dispensed an average of two antihypertensive medicines, with ACE inhibitors/ARBs most frequently dispensed. One quarter of our cohort who developed aggravated hypertension received dose intensification and these patients were dispensed an average of three antihypertensive agents after aggravation. Patients experiencing aggravated hypertension were predominantly dispensed ACE inhibitors, ARBs, and CCBs at baseline and their use of diuretics increased after aggravation. In US clinical data, a greater proportion of patients with pre-existing hypertension developed a hypertensive response (55\% vs $40 \%$ ) and pre-existing hypertension was a risk factor for VSPi-induced hypertension [15]. However, we found no significant difference between these two patient subgroups in our study, indicating an index of suspicion is warranted regardless of blood pressure history.

Consistent with US data [15], older age ( $\geq 60$ years) and use of an oral TKI agent compared to bevacizumab were risk factors for new-onset hypertension after VSPi use. Only cancer type was associated with aggravation of hypertension in our cohort, with RCC patients displaying the greatest risk. This is consistent with prior evidence [11,33,34], and may be due to higher VEGF levels in RCC compared to other cancer patients, or it may be related to prior nephrectomy or reduced renal function [11, 34]. Alternatively, this may reflect information bias because we could only observe treated hypertension and those with RCC may be more likely to receive antihypertensive therapy.

The major limitation of our study is the lack of clinical information in the PBS database, noting that some antihypertensive medicines are prescribed for other indications. Thus, our data may over-estimate the incidence of clinically treated hypertension. We also could not describe cancer stage, related clinical and laboratory data, or detailed blood pressure measures, nor could we conduct risk factor analyses 
incorporating these factors. We did not have diagnoses data and ascertained hypertension through dispensing records. PBS records do not contain data for medicines dispensed to public hospital inpatients and we only observed anti-hypertensive medications dispensed in the community, so our study may over-estimate the time to first dispensed anti-hypertensive medication. Despite these limitations, our study covers the entire Australian population and both small molecule TKIs and antibody VSPi.

Our study provides real-world estimates of the incidence of, and risk factors for, VSPi-induced hypertension in a whole-of-population setting. Despite the well-known risks of cardiac adverse events associated with VSPi, there is little existing real-world evidence on hypertension incidence, risk factors and management in VSPi-treated patients. Our findings suggest that the real-world incidence of VSPiinduced hypertension is similar to that observed in pivotal clinical trials and they add valuable data for patients receiving care in routine clinical practice. Australian antihypertensive prescribing data thus appears to be a reliable and cost-effective proxy for clinically identified hypertension in this at-risk population.

\section{Declarations}

\section{Acknowledgments}

We thank the Services Australia for providing the data and Melisa Litchfield for extracting the study sample and providing analytical advice.

\section{Author's contributions}

SH, BD, SAP and CMV conceived and designed the study. SH performed the data analysis. SH, BD, SAP, $M T V L$ and $C M V$ interpreted the data, drafted the paper and approved the final version of the paper.

\section{Funding}

This research is supported by a Cooperative Research Centre Project (CRC-P) Grant from the Australian Government Department of Industry, Innovation and Science (ID: CRC-P-439), internal funding from UNSW Sydney and an NHMRC Postdoctoral Fellowship to Marina van Leeuwen (ID1012141).

\section{Data availability}

The datasets used in this study are not available for sharing, however, the data can be requested from Services Australia.

\section{Ethics approval}

This study was approved by the New South Wales Population and Health Services Research Ethics Committee (Reference 2013/11/494). The requirement for informed consent was waived because the investigators did not receive personally identifying information. Data access was granted by the Services Australia External Request Evaluation Committee (Approval Number: RMS0825). 


\section{Conflict of interest}

Sallie-Anne Pearson is a member of the Drug Utilisation Sub-Committee of the Pharmaceutical Benefits Advisory Committee. The views expressed in this paper do not represent those of either Committee.

The Centre for Big Data Research in Health, UNSW Sydney has received funding from AbbVie Australia to conduct research unrelated to the present study. AbbVie did not have any knowledge of, or involvement in, the present study.

The remaining authors declare no competing interests.

\section{Consent for publication}

Not applicable.

\section{References}

1. Hicklin DJ, Ellis LM. (2005). Role of the Vascular Endothelial Growth Factor Pathway in Tumor Growth and Angiogenesis. 23, 1011-27. 10.1200/jco.2005.06.081.

2. Dreyfus B, Kawabata H, Gomez A. Selected adverse events in cancer patients treated with vascular endothelial growth factor inhibitors. Cancer Epidemiol. 2013;37:191-6. 10.1016/j.canep.2012.11.001.

3. Jayson GC, Kerbel R, Ellis LM, Harris AL. Antiangiogenic therapy in oncology: current status and future directions. The Lancet. 2016;388:518-29. 10.1016/s0140-6736(15)01088-0.

4. Mouhayar E, Durand J-B, Cortes J. Cardiovascular toxicity of tyrosine kinase inhibitors. Expert Opinion on Drug Safety. 2013;12:687-96. 10.1517/14740338.2013.788642.

5. Touyz RM, Herrmann J. (2018). Cardiotoxicity with vascular endothelial growth factor inhibitor therapy. NPJ Precis Oncol 2, 13. 10.1038/s41698-018-0056-z.

6. Agarwal M, Thareja N, Benjamin M, Akhondi A, Mitchell GD. Tyrosine Kinase Inhibitor-Induced Hypertension. Curr Oncol Rep. 2018;20:65. 10.1007/s11912-018-0708-8.

7. Cohen JB, Geara AS, Hogan JJ, Townsend RR. Hypertension in Cancer Patients and Survivors: Epidemiology, Diagnosis, and Management. JACC CardioOncol. 2019;1:238-51. 10.1016/j.jaccao.2019.11.009.

8. Chen J, Lu Y, Zheng Y. Incidence and risk of hypertension with bevacizumab in non-small-cell lung cancer patients: a meta-analysis of randomized controlled trials. Drug Des Devel Ther. 2015;9:475160. 10.2147/dddt.S87258.

9. Nakaya A, Kurata T, Yokoi T, Iwamoto S, Torii Y, Katashiba Y, et al. Retrospective analysis of bevacizumab-induced hypertension and clinical outcome in patients with colorectal cancer and lung cancer. Cancer Med. 2016;5:1381-7. 10.1002/cam4.701. 
10. Ranpura V, Pulipati B, Chu D, Zhu X, Wu S. Increased risk of high-grade hypertension with bevacizumab in cancer patients: a meta-analysis. Am J Hypertens. 2010;23:460-8. 10.1038/ajh.2010.25.

11. Zhu X, Stergiopoulos K, Wu S. Risk of hypertension and renal dysfunction with an angiogenesis inhibitor sunitinib: systematic review and meta-analysis. Acta Oncol. 2009;48:9-17. 10.1080/02841860802314720.

12. Zhu C, Ma X, Hu Y, Guo L, Chen B, Shen K, et al. Safety and efficacy profile of lenvatinib in cancer therapy: a systematic review and meta-analysis. Oncotarget. 2016;7:44545-57. 10.18632/oncotarget.10019.

13. Izzedine H, Ederhy S, Goldwasser F, Soria JC, Milano G, Cohen A, et al. Management of hypertension in angiogenesis inhibitor-treated patients. Ann Oncol. 2009;20:807-15. 10.1093/annonc/mdn713.

14. Thompson LA, Saseen JJ, O'Bryant CL, Allen RR, Nair KV. Claims analysis of hypertension occurrence, severity changes and patterns of antihypertensive use in cancer patients receiving vascular endothelial growth factor inhibitors. J Oncol Pharm Pract. 2015;21:258-67. $10.1177 / 1078155214530177$.

15. Hamnvik OP, Choueiri TK, Turchin A, McKay RR, Goyal L, Davis M, et al. Clinical risk factors for the development of hypertension in patients treated with inhibitors of the VEGF signaling pathway. Cancer. 2015;121:311-9. 10.1002/cncr.28972.

16. Mellish L, Karanges EA, Litchfield MJ, Schaffer AL, Blanch B, Daniels BJ, et al. The Australian Pharmaceutical Benefits Scheme data collection: a practical guide for researchers. BMC Res Notes. 2015;8:634. 10.1186/s13104-015-1616-8.

17. Schaffer AL, Buckley NA, Dobbins TA, Banks E, Pearson SA. The crux of the matter: Did the ABC's Catalyst program change statin use in Australia? Med J Aust. 2015;202:591-5. 10.5694/mja15.00103.

18. Australian Government Department of Health. The Pharmaceutical Benefit Scheme. Available at: http://www.pbs.gov.au/pbs/home. Accessed 15/05, 2020.

19. Schaffer AL, Pearson SA, Buckley NA. How does prescribing for antihypertensive products stack up against guideline recommendations? An Australian population-based study (2006-2014). Br J Clin Pharmacol. 2016;82:1134-45. 10.1111/bcp.13043.

20. Pratt NL, Kerr M, Barratt JD, Kemp-Casey A, Kalisch Ellett LM, Ramsay E, et al. The validity of the RxRisk Comorbidity Index using medicines mapped to the Anatomical Therapeutic Chemical (ATC) Classification System. BMJ Open. 2018;8:e021122. 10.1136/bmjopen-2017-021122.

21. Mealing NM, Dobbins TA, Pearson SA. Validation and application of a death proxy in adult cancer patients. Pharmacoepidemiol Drug Saf. 2012;21:742-8. 10.1002/pds.2257.

22. Pintilie M. Competing Risks: A Practical Perspective. Hoboken: John Wiley \& Sons, Incorporated;; 2006.

23. Funakoshi T, Latif A, Galsky MD. Risk of hypertension in cancer patients treated with sorafenib: an updated systematic review and meta-analysis. J Hum Hypertens. 2013;27:601-11. 
10.1038/jhh.2013.30.

24. Qi WX, He AN, Shen Z, Yao Y. Incidence and risk of hypertension with a novel multi-targeted kinase inhibitor axitinib in cancer patients: a systematic review and meta-analysis. Br J Clin Pharmacol. 2013;76:348-57. 10.1111/bcp.12149.

25. Qi WX, Lin F, Sun YJ, Tang LN, He AN, Yao Y, et al. Incidence and risk of hypertension with pazopanib in patients with cancer: a meta-analysis. Cancer Chemother Pharmacol. 2013;71:431-9.

10.1007/s00280-012-2025-5.

26. Kennedy-Martin T, Curtis S, Faries D, Robinson S, Johnston J. A literature review on the representativeness of randomized controlled trial samples and implications for the external validity of trial results. Trials. 2015;16:495. 10.1186/s13063-015-1023-4.

27. Harding JL, Sooriyakumaran M, Anstey KJ, Adams R, Balkau B, Brennan-Olsen S, et al. Hypertension, antihypertensive treatment and cancer incidence and mortality: a pooled collaborative analysis of 12 Australian and New Zealand cohorts. J Hypertens. 2016;34:149-55.

10.1097/hjh.0000000000000770.

28. Azizi M, Chedid A, Oudard S. (2008). Home Blood-Pressure Monitoring in Patients Receiving Sunitinib. 358, 95-7. 10.1056/NEJMc072330.

29. Maitland ML, Kasza KE, Karrison T, Moshier K, Sit L, Black HR, et al. Ambulatory monitoring detects sorafenib-induced blood pressure elevations on the first day of treatment. Clin Cancer Res. 2009;15:6250-7. 10.1158/1078-0432.Ccr-09-0058.

30. Curigliano G, Cardinale D, Suter T, Plataniotis G, de Azambuja E, Sandri MT, et al. Cardiovascular toxicity induced by chemotherapy, targeted agents and radiotherapy: ESMO Clinical Practice Guidelines. Ann Oncol. 2012;23(Suppl 7):vii155-66. 10.1093/annonc/mds293.

31. Wasserstrum Y, Kornowski R, Raanani P, Leader A, Pasvolsky O, lakobishvili Z. (2015). Hypertension in cancer patients treated with anti-angiogenic based regimens. Cardio-Oncology 1. 10.1186/s40959015-0009-4.

32. Versmissen J, Mirabito Colafella KM, Koolen SLW, Danser AHJ. Vascular Cardio-Oncology: Vascular Endothelial Growth Factor inhibitors and hypertension. Cardiovasc Res. 2019;115:904-14. $10.1093 / \mathrm{cvr} / \mathrm{cvz} 022$.

33. Yang X, Pan X, Cheng X, Kuang Y, Cheng Y. Risk of Hypertension With Sorafenib Use in Patients With Cancer: A Meta-Analysis From 20,494 Patients. Am J Ther. 2017;24:e81-101.

10.1097/mjt.0000000000000331.

34. Li Y, Li S, Zhu Y, Liang X, Meng H, Chen J, et al. Incidence and risk of sorafenib-induced hypertension: a systematic review and meta-analysis. J Clin Hypertens (Greenwich). 2014;16:177-85.

10.1111/jch.12273.

\section{Figures}




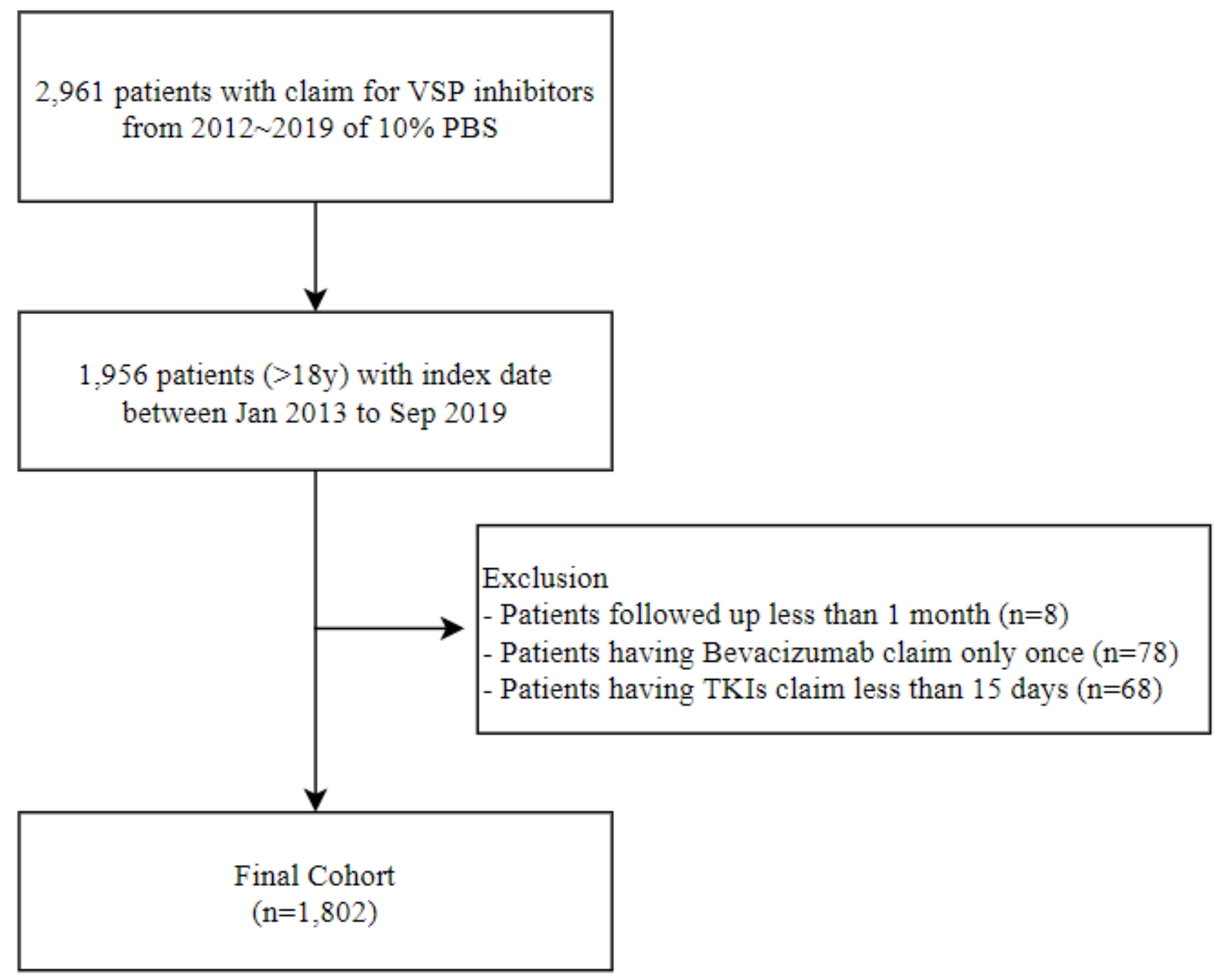

Figure 1

Consort flow diagram of study population 


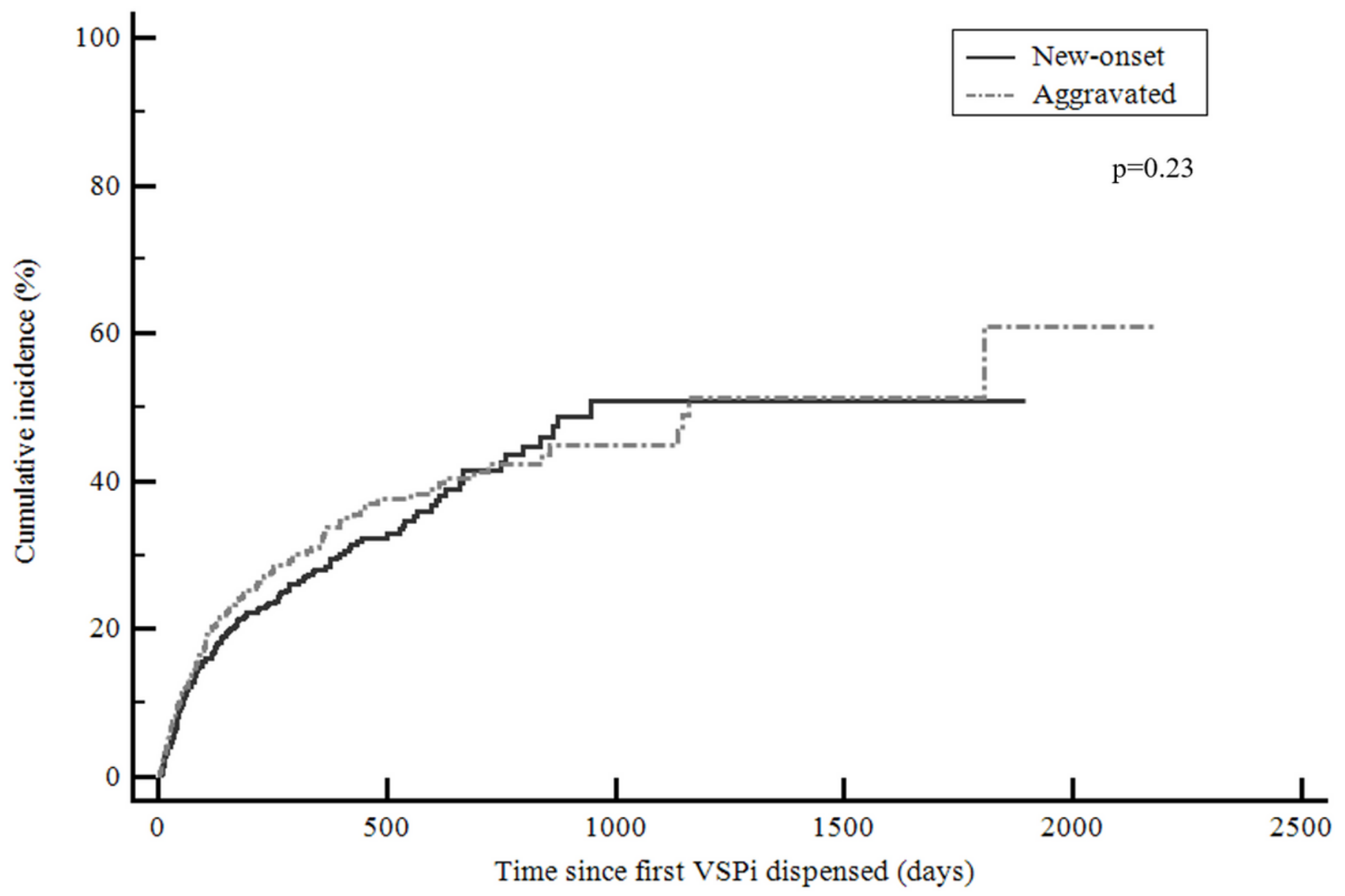

Figure 2

Cumulative incidence of hypertension therapy in Australians dispensed VSPi 